\title{
Double peak structure and diamagnetic wings of the magnetotail current sheet
}

\author{
G. Zimbardo ${ }^{1, *}$, A. Greco ${ }^{1, *}$, P. Veltri ${ }^{1, *}$, A. L. Taktakishvili ${ }^{2}$, and L. M. Zelenyi ${ }^{3}$ \\ ${ }^{1}$ Physics Department, University of Calabria, Ponte P. Bucci, Cubo 31C, I-87036 Rende, Italy \\ ${ }^{2}$ Abastumani Astrophysical Observatory, A. Kazbegi str., 2a, 380060, Tbilisi, Georgia \\ ${ }^{3}$ Space Research Institute, Profsoyuznaya 84/32, 117810 Moscow, Russia \\ *Also at Istituto Nazionale di Fisica della Materia, Unita' di Cosenza, Italy
}

Received: 2 October 2003 - Revised: 10 March 2004 - Accepted: 20 April 2004 - Published: 14 July 2004

Part of Special Issue "Spatio-temporal analysis and multipoint measurements in space"

\begin{abstract}
Recent Cluster observations in the magnetotail at about 20 Earth radii downtail have unambiguously shown that sometimes the current sheet is bifurcated, i.e. it is divided in two layers. We report numerical simulations of the ion dynamics in a quasi-neutral sheet in the presence of magnetic turbulence, which is often observed in the magnetotail, and for various anisotropies of the ion distribution function. Ions are injected at the boundary of the simulation box with a velocity distribution corresponding to a shifted Maxwellian. The simulation parameters, are adjusted to be similar to those of Cluster observations. We find that even for moderate fluctuation levels, the computed current density profile develops a double peak, in agreement with the observations. By varying the anisotropy of the injected distribution function, we are able to reproduce, for weak anisotropy, the magnetic field overshoots which are sometimes observed prior to magnetotail traversals. Therefore, we suggest an ion current profile with a double peak due to magnetic turbulence, and with possible diamagnetic current wings, present in the case of weak anisotropy of the ion distribution function.
\end{abstract}

Key words. Magnetospheric physics (magnetotail; plasma sheet) - Space plasma physics (numerical simulation studies)

\section{Introduction}

Recent Cluster observations in the magnetotail at about 20 Earth radii downtail have unambiguously shown that sometimes the current sheet is bifurcated, i.e. it is divided in two layers, with a corresponding flattening of the magnetic field profile in the central sheet (Nakamura et al., 2002; Runov et al., 2003; Sergeev et al., 2003). These bifurcated

Correspondence to: G. Zimbardo

(zimbardo@fis.unical.it) current sheets appear to be not necessarily associated with magnetic reconnection, that is with dipolarization or fast flows. Previously, current density profiles with a double peak were observed in the near-Earth tail by ISEE 1 and 2 (Sergeev et al., 1993), and were deduced by a statistical analysis of Geotail data in the distant tail (Hoshino et al., 1996). Evidence of double peaked current sheets in the near-Earth tail were recently reported by Geotail (Asano et al., 2003), too. These observations have stimulated a number of studies aimed at understanding the origin of the double peaked current profile. Asano et al. (2003) have considered that a Hall electric field normal to the sheet develops in thin current sheets because the ion motion is unmagnetized while the electron motion is magnetized. Using the GSM coordinate system, an $E_{z} \times B_{x}$ motion in the $y$ direction (dawnward) is induced, which is most important for magnetized electrons away from the central part of the current sheet. In such a case, the double current peak would be supported by electrons. Also, in numerical simulations of magnetic reconnection, a squeezed-X current pattern is usually found around the reconnection site, so that a crossing of the current structure at some distance of the $\mathrm{X}$ neutral line would give a double current peak. In hybrid simulations (particle ions plus fluid electrons) it is found that this current is supported mostly by electrons (e.g. Hesse et al. (1996); Arzner et al. (2001)).

On the other hand, the Cluster current sheet crossings of 29 August 2001 (Runov et al., 2003, 2004) and of 26 September 2001 (Sergeev et al., 2003) appear to be not closely associated with strong reconnection events, but rather with a large-scale, wave-like flapping motion of the current sheet as a whole. Although during the 26 September crossing no particular feature in the duskward ion velocity $V_{y}$ is observed, suggesting that the bifurcated current is supported by electrons (Sergeev et al., 2003), during the 29 August crossing a strong duskward ion velocity is observed at about 10:55 UT, 
with bulk velocities up to $V_{y} \sim 400 \mathrm{~km} / \mathrm{s}$ (Runov et al., 2004), suggesting that the current is supported by the ions. In this connection, we notice that a number of current sheet equilibria based on current carrying ions have been developed. By varying the drift velocity $v_{D}$ of the transient ion distribution function (see later), Holland and Chen (1993) obtained selfconsistent solutions with a thin current sheet embedded in a thicker plasma sheet. Thin current sheets with double peaks from about 11 to $14 R_{E}$ downtail were obtained by Delcourt and Belmont (1998) by means of test particles evolving in the Tsyganenko 89 magnetic field model. In that study, the current density double peak was due to the onset of ion meandering motion with larger $v_{y}$ at the upper and lower part of the trajectory. By making use of the quasi-adiabatic invariant of motion $I_{z}=(2 \pi)^{-1} \oint m v_{z} d z$ (Büchner and Zelenyi, 1989), and considering anisotropic ion distribution functions, a series of self-consistent Vlasov current sheet structures were developed by Kropotkin et al. (1997); Sitnov et al. (2000); Malova et al. (2000); Zelenyi et al. (2000). Solutions were obtained which also describe the magnetic overshoots which are associated with the diamagnetic current wings (Holland and Chen, 1993; Malova et al., 2000; Zelenyi et al., 2000). Indeed, Cluster data of the 26 September crossing are particularly stimulating because the magnetic overshoots are observed (i) by more than one spacecraft, (ii) repeatedly by a single spacecraft, and (iii) on both sides of the current sheet. Malova et al. (2000) and Zelenyi et al. (2002a) considered the presence of a trapped plasma component, and found that for weak anisotropy (small $v_{D}$ ) of the transient ions, the diamagnetic wings are suppressed when the number of trapped particles inside the current sheet is increased. Also, increasing the trapped plasma population, the current in the center of the sheet decreases and a double peak is obtained ( $\mathrm{Ze}$ lenyi et al., 2002a). The effects of the nonadiabatic jumps of the quasi-adiabatic invariant $I_{z}$, leading to the formation of a trapped ion population, double peaked current profile, and eventual current sheet break up, were considered in Zelenyi et al. (2002b). More recently, Sitnov et al. (2003) extended the analysis of self-consistent Vlasov equilibria to the case of ion temperature anisotropy. They obtained bifurcated current sheets in the cases $T_{\perp}>T_{\|}$(where $T_{\perp}\left(T_{\|}\right)$is the ion temperature perpendicular (parallel) to the magnetic field), together with relatively flat current density profiles, which compare rather well with Cluster observations.

On the other hand, the observations of ISEE 2, AMPTE/IRM, Geotail, Interball, and Cluster spacecraft have shown the existence of plasma and magnetic field turbulence in the distant and near-Earth's magnetotail (Angelopoulos et al., 1993; Hoshino et al., 1994; Ohtani et al., 1995; Bauer et al., 1995; Borovsky et al., 1997; Voros et al., 2003). Usually, the turbulence is stronger in the center of the plasma sheet (Bauer et al., 1995; Hoshino et al., 1994), while a more regular magnetic configuration is found in the vicinity of the magnetospheric lobes. Sergeev et al. (1993) already pointed out that a double peaked current density profile is observed in the same periods when magnetic turbulence is present. The influence of turbulence on the current sheet structure in the distant magnetotail, where the average component of the magnetic field normal to the current sheet $B_{n}$ is negligible, was considered by Veltri et al. (1998) by means of a test particle simulation. They found that for fluctuation levels $\delta B / B_{0}>0.2$ the current sheet has a double layer structure, with a current density minimum in the center of the neutral sheet. Recently, Greco et al. (2002); Zimbardo et al. (2003) considered the near-Earth magnetotail, where $B_{n}>0$, and found that even in this case the magnetic fluctuations induce a double layer structure in the current sheet.

Clearly, there are several possibilities to interpret the observations of bifurcated current sheets, and it is possible that different scenarios and different current carriers apply in different cases (we note, for instance, that in the Geotail current sheet crossing of 23 April 1996, the dawn-to-dusk current was mostly carried by ions before 10:03 UT, and mostly by electrons after 10:03 UT (Asano et al., 2003). In this paper we extend the work of Greco et al. (2002); Zimbardo et al. (2003), by tailoring the simulation parameters to the Cluster observations during the magnetotail crossings which exhibited a bifurcated current sheet. We consider moderate turbulence levels, and we pay attention to the anisotropy of the injected ion distribution function, that is to the ratio $\epsilon=v_{t h} / v_{D}$ between the thermal velocity $v_{t h}$ and the drift velocity of ions along the average magnetic field $v_{D}$. We find that $\epsilon$ also influences the current sheet profile: for sufficient fluctuation levels the double current peak is formed, while moderately large values of $\epsilon$ lead to the formation of diamagnetic (negative) current wings, as predicted by self-consistent kinetic studies in the absence of turbulence (Holland and Chen, 1993; Malova et al., 2000; Zelenyi et al., 2000). Conversely, for small values of $\epsilon$ the negative current wings disappear.

\section{Numerical Model}

The magnetic field model used in the simulation is described in full detail in Veltri et al. (1998); Greco et al. (2002); Zimbardo et al. (2003). For completeness, we recall here some of the main features: The considered magnetic field configuration consists of an unperturbed, sign reversing component, directed along the Earth-Sun axis, $\mathbf{B}_{0 x}(z)=B_{0 x}(z) \mathbf{e}_{x}$; an unperturbed, constant, normal component $\mathbf{B}_{n}=B_{n} \mathbf{e}_{z}$; and of three-dimensional stationary magnetic fluctuations $\delta \mathbf{B}(\mathbf{r})$ having a power law spectrum. We use for $B_{0 x}(z)$ the expression of a modified Harris magnetic field reversal (Veltri et al., 1998)

$B_{0 x}(z) \simeq B_{0} \tanh (z / \lambda)$.

Here, $\lambda=0.25 L$ is the current sheet half thickness, and $L$ is the thickness of the considered magnetic field configuration (i.e. of the simulation box).

The power law spectrum of the magnetic fluctuations, regularly observed by satellites, is represented as the sum of 
static magnetic perturbations (Veltri et al., 1998; Greco et al., 2002)

$\delta \mathbf{B}(\mathbf{r})=\sum_{k, \sigma} \delta B(\mathbf{k}) \mathbf{e}_{\sigma}(\mathbf{k}) \exp \left(i\left(\mathbf{k} \cdot \mathbf{r}+\phi_{\mathbf{k}}^{\sigma}\right)\right)$,

where $\mathbf{e}_{\sigma}(\mathbf{k})$ are the polarization unit vectors (Veltri et al., 1998), $\phi_{\mathbf{k}}^{\sigma}$ are random phases, and where

$\delta B(\mathbf{k})=\frac{C}{\left(k_{x}{ }^{2} l_{x}{ }^{2}+k_{y}{ }^{2} l_{y}{ }^{2}+k_{z}{ }^{2} l_{z}{ }^{2}+1\right)^{\alpha / 4+1 / 2}}$,

where $C$ is a normalization constant, and $l_{x}, l_{y}, l_{z}$ are the turbulence correlation lengths in the $x, y, z$ directions, respectively. By a proper choice of random phases, fluctuations are made stronger at $z \simeq 0$, where they sum up coherently (Veltri et al., 1998). The correlation lengths are fixed in terms of the thickness of the simulation box as $l_{x}=l_{y}=0.25 \mathrm{~L}$, $l_{z}=0.05 \mathrm{~L}$, in order to mimic the geometry of the magnetotail (i.e. $\left.l_{z} \ll l_{x}, l_{y}\right)$. Note that the above expression for $\delta B(\mathbf{k})$ yields a spectral index $\alpha$ for the turbulence spectral power density $d W / d k=\frac{1}{2}(\delta B)^{2} k^{2} \propto k^{-\alpha}$. For the nearEarth magnetotail $\alpha=2.3$ may be appropriate (Slavin et al., 1985; Ohtani et al., 1995; Borovsky et al., 1997; Zelenyi et al., 1998). In addition to the above magnetic field configuration, we consider a constant cross tail electric field, oriented in the dawn to dusk direction, $\mathbf{E}=E_{y} \mathbf{e}_{y}$. We note that in the presence of three-dimensional magnetic fluctuations, the dawn-dusk electric field cannot be removed by transforming to the de Hoffman-Teller frame.

In this paper we consider the electrons as a charge neutralizing background, and leave a more detailed study of their influence for future work. Although we are considering static magnetic perturbations, it can be shown that the fluctuating electric field (produced by realistic magnetic perturbations) has a negligible impact on the bulk properties of particles (Greco et al., 2002). The equations of motion can be written in a dimensionless form by normalizing all length scales to the unit length $L$, velocities to electric drift velocity $V_{E}=c E_{y} / B_{0}$, magnetic and electric fields to $B_{0}$ and $E_{y}$, respectively, and time to ion gyrofrequency $\omega_{0}=e B_{0} / m_{i} c$. Typical values of the normalization parameters for the nearEarth magnetotail can be obtained by setting $B_{0}=10 \mathrm{nT}$ and $E_{y}=0.1 \mathrm{mV} / \mathrm{m}$. The unit length may be assumed to be $L=10^{4} \mathrm{~km}$, which implies a current sheet half thickness $\lambda=0.25 L=2500 \mathrm{~km}$. We also define $\delta B=\sqrt{\langle\delta \mathbf{B} \cdot \delta \mathbf{B}\rangle}$, with the average made over the simulation box , and $\delta b \equiv \delta B / B_{0}$ and $b_{n} \equiv B_{n} / B_{0}$.

The particles are injected with random positions in the $x y$ plane, and to some distance from the upper and lower boundaries of the simulation box, at $z= \pm 0.7 \mathrm{~L}$, in a buffer layer of proper thickness to avoid the influence of the boundaries on the results. The particle velocities at injection are distributed according to a drifting Maxwellian

$f\left(v_{\|}, v_{\perp}\right) \propto \exp \left(-\frac{\left(v_{\|}-v_{D}\right)^{2}+v_{\perp}^{2}}{2 v_{t h}^{2}}\right)$.

Here $v_{D}$ is the streaming velocity along the unperturbed magnetic field, and $v_{t h}$ is the thermal velocity. Note that $\epsilon=v_{t h} / v_{D}>1$ implies that the thermal velocity is larger than the streaming velocity, so that $v_{\perp}$ and $v_{\|}$will be of the same order of magnitude, corresponding to weak anisotropy. On the other hand, for $\epsilon<1$ the thermal velocity is smaller than the streaming velocity, so that $v_{\|}>v_{\perp}$, corresponding to strong anisotropy and, for $\epsilon \ll 1$, to field-aligned distributions.

\section{Numerical Results}

In the present simulations, we assume a value of the normal magnetic field of $b_{n}=0.02$, which corresponds well to the Cluster observations of 29 August 2001 (Runov et al., 2004) and of 26 September 2001 (Sergeev et al., 2003) magnetotail crossings. We assume that $v_{t h}=400 \mathrm{~km} / \mathrm{s}$, corresponding to a particle energy of about $2 \mathrm{keV}$, which corresponds to the ion temperature observed by Cluster at the boundary of the current sheet (Runov et al., 2004). At injection, the ion Larmor radius in the asymptotic field $B_{0}$ is $\rho_{0} \simeq 0.05 \mathrm{~L}$, so that $\rho_{0} \simeq \lambda / 5$. Thus, our parameters correspond to a relatively thin current sheet. The dawn-dusk electric field exhibited relatively small, and sometimes negative, values during the observations of bifurcated current sheets (data from CDAWeb; data provider G. Gustafsson at IRFU): accordingly, a value of $E_{y}=0.05 \mathrm{mV} / \mathrm{m}$ has been used. During the Cluster magnetotail crossings, the magnetic turbulence was at moderate levels, being largest for the 12 August traversal (Nakamura et al., 2002) and smallest for the 26 September (Sergeev et al., 2003). A value of $\delta b$ of the order of 0.1 is indicated by Runov et al. (2004) for the 29 August crossing. Thus, we assume turbulence levels $\delta b$ ranging from 0.1 to 0.3 (the turbulence level is adjusted by varying the constant $C$ in Eq. (3)).

For each given set of parameters, we inject many particles in the simulation box and numerically integrate their equation of motion. Then we compute the distribution function moments as density $n$, bulk velocity $\mathbf{V}$, current density $\mathbf{j}$, temperature $T$, etc. on a three-dimensional grid. Typically, 80000 particles are injected for each run. In a test particle simulation, the normalization for the number density is, to a good extent, arbitrary. Here, it is based on the consideration that the ion current $I_{y}$ has to be strong enough to reproduce the unperturbed magnetic field $B_{0 x}(z)$ introduced in our model. Indeed, Ampere's law leads to $2 B_{0} L_{x}=4 \pi I_{y} / c$, with $I_{y}=\int j_{y} d x d z$ the total current across a section of the current sheet of length $L_{x}$. On the other hand, velocity is expressed in units of $V_{E}$, so that the normalization for density is obtained as $n^{*}=I_{y} /\left[e \int V_{y} d x d z\right]$, with $I_{y}$ constant for all the runs (this implies that when the average value of $V_{y}$ is large, the density is low). The simulation results for the $z$ profiles (averaged over $x$ and $y$ ) of the ion density $n$, the velocity $V_{y}$, the current density $j_{y}$ and the corresponding magnetic field profile, obtained as $B_{x}(z)=\int_{0}^{z} j_{y}\left(z^{\prime}\right) d z^{\prime}$, are shown in Fig. 1 for various turbulence levels $\delta b$ and for weak anisotropy, $\epsilon=4$. Note that relatively flat plasma density profiles are obtained, in agreement with Cluster observations (Sergeev et al., 2003; Runov et al., 2004), while the current 


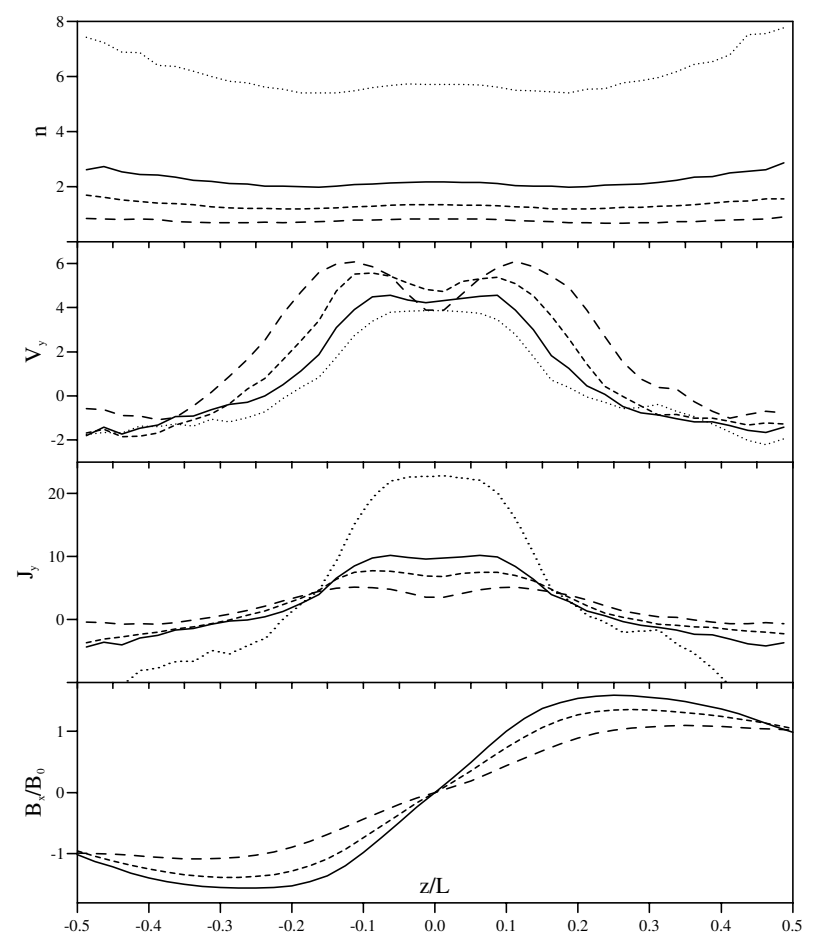

Fig. 1. Simulation results for the plasma density (top), ion velocity (middle top), current density (middle bottom), and corresponding magnetic field profile (bottom), for $b_{n}=0.02, \epsilon=4$, and for different values of the turbulence level: $\delta b=0.12$ (dotted line), $\delta b=0.15$ (solid line), $\delta b=0.2$ (short dashed line), $\delta b=0.3$ (long dashed line). The magnetic field for $\delta b=0.12$ is not reported, as it is mostly out of scale.

density profiles correspond to a thin current sheet enbedded in a thicker plasma sheet. The plasma density appears to be slightly larger toward the boundary of the simulation box because of the bouncing motion (oscillating $z$ ) of the ions, which implies larger $v_{z}$ in the center; this effect is decreased when increasing $\delta b$, as turbulence slows down the bouncing motion. The influence of magnetic turbulence on the velocity $V_{y}$ is twofold: on the one hand, turbulence causes the velocity at $z \simeq 0$ to decrease because it is an obstacle to meandering motion, as it scatters the ions around. On the other hand, the velocity out of the central region increases because of the presence of particles scattered away from the center, and because the fluctuations $\delta B_{y}$ also bend the field lines in the $y$ direction. This allows for cross field motion (with respect to the average field) even for magnetized particles, although of the biased diffusive type since $\left\langle\delta B_{y}\right\rangle=0$ and $E_{y} \neq 0$. Increasing $\delta b$, the current carrying region widens, and both $j_{y}$ and $V_{y}$ exhibit a marked double layer structure. This structure is due to the enhanced braking at $z \simeq 0$ due to the concentration of turbulence in the quasi-neutral sheet, which models the observations (Bauer et al., 1995). Well developed, negative current wings are obtained for all the runs of Fig. 1, due to the weak anisotropy of the ion distribution function $(\epsilon=4)$. The corresponding magnetic overshoots, $\left|B_{x}\right|>B_{0}$, are found in the $B_{x}$ profile. Increasing the turbulence level, the scattering of ions away from the central region tends to decrease

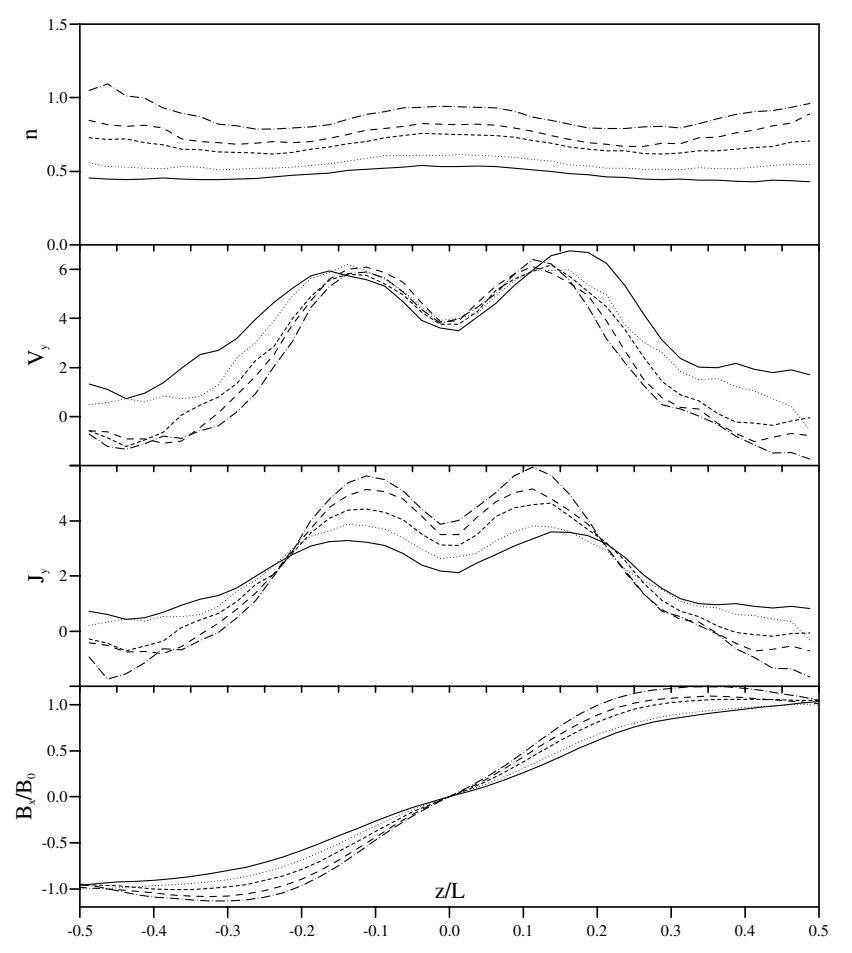

Fig. 2. Same as Fig. 1 but for $\delta b=0.3$ and $b_{n}=0.02$, and for different values of the anisotropy parameter $\epsilon=v_{t h} / v_{D}: \epsilon=0.8$ (solid line), $\epsilon=1$ (dotted line), $\epsilon=2$ (short dashed line), $\epsilon=4$ (long dashed line), $\epsilon=8$ (dash-dotted line).

the extent of the diamagnetic current wings (an effects similar to that due to the presence of a trapped ion population, see Zelenyi et al. (2002a)). Indeed, these wings were already obtained by Greco et al. (2002) for zero to low $(\delta b=0.1)$ turbulence levels (in those runs, $\epsilon=0.85$ ), but when $\delta b$ was increased the current sheet was inflated, and the paramagnetic current $j_{y}>0$ was dominating on the diamagnetic current.

Figure 2 shows the same quantities for a fixed value of $\delta b=0.3$ and for $\epsilon=0.8,1,2,4,8$. It can be seen that a neat double current layer is obtained for all the values of $\epsilon$. Beside the bifurcated current layer, Runov et al. (2004) were able to show that the ion velocity $V_{y}$ is smaller in the central part of the current sheet than in the out of central part, a feature which is also found in Figs. 1 and 2. For $\epsilon=2,4,8$ (weak anisotropy), negative current density wings appear, while they are not found for $\epsilon=0.8,1$. Correspondingly, magnetic field overshoots are obtained in the computed magnetic field profile. In Fig. 2 we can see that the larger $\epsilon$ is, the higher the positive current peaks. However, this is an effect of the normalization adopted for the current density (i.e. for the ion density). Since we impose that the total current $I_{y}$ be constant, the presence of negative current wings for $\epsilon=2,4,8$ leads to larger values of the positive current density, too. Still, the ratio between the peak height and the central valley height in Fig. 2 is almost constant; the velocity panel shows that in the central part the ion velocity changes little with $\epsilon$. 
This indicates that the ion dynamics in the central current sheet is not very sensitive to the anisotropy at injection, because of the enhanced pitch angle scattering due to magnetic turbulence. Further, we can imagine that magnetic fluctuations allow ions to populate the phase space which would belong (in the absence of turbulence) to trapped particles, and we recall that the presence of a substantial trapped plasma population allowed Zelenyi et al. (2002a) to obtain a double peaked current density profile. One might wonder whether the effect of turbulence is just to create trapped ions, which, in turn, lead to a bifurcated current structure; however, the influence of trapped ions was found to be significant only in cases of weak anisotropy (Malova et al., 2000; Zelenyi et al., 2002a), while Fig. 2 shows that we obtain a double peaked current profile for all values of $\epsilon$. Hence, the influence of turbulence is not simply pitch angle scattering, but comprises a diffusive slowing of ordered motion. Clearly, the scenario is different in the absence of turbulence, when the whole current profile depends on the anisotropy $\epsilon$ and on the way the current sheet is populated (Holland and Chen, 1993; Zelenyi et al., 2000, 2002a; Sitnov et al., 2003).

In comparison to our previous works, we note that in the present runs the ion temperature at injection is assumed to be $2 \mathrm{keV}$, while in Greco et al. (2002); Zimbardo et al. (2003) it was $0.3 \mathrm{keV}$. This implies that the current sheet thickness is increased (we have $\rho_{0} \simeq \lambda / 5$ rather than $\rho_{0} \simeq \lambda / 12$ ) and that the separation of the current density peaks is larger with respect to those reported by Greco et al. (2002). Inspection of Fig. 2 shows that the current density maxima are located very close to the values of $z / L$ where $\left|B_{x}\right| \simeq B_{0} / 2$, as in Cluster observations (Sergeev et al., 2003; Runov et al., 2004).

The above results show that it is possible to reproduce both the double peak of the current density and the diamagnetic current wings, as observed by Cluster (Nakamura et al., 2002; Runov et al., 2003; Sergeev et al., 2003).

\section{Conclusions}

We have studied by a test particle simulation the ion dynamics in the magnetotail in the presence of a stationary magnetic turbulence $\delta B$. The magnetic turbulence observed by several spacecraft was modeled numerically, and a simulation was performed in which ions are injected into the quasi-neutral current sheet with different anisotropies. Cluster data taken during the current sheet crossings of 29 August 2001, and 26 September 2001, were used to set simulation parameters like the ion temperature, the dawn-dusk electric field, the ratio $B_{n} / B_{0}$, and the fluctuation level $\delta B / B_{0}$. For a moderate level of fluctuations, $\delta B / B_{0}>\sim 0.15$, the current density profile exhibits a double peak structure. We are also able to reproduce a plasma sheet thicker than the current sheet, the enhanced $V_{y}$ out of the central region (Runov et al., 2004), and the position of the current density maxima corresponding to $\left|B_{x}\right| \simeq B_{0} / 2$. Our results agree well with previous (Sergeev et al., 1993; Hoshino et al., 1996) and recent (Nakamura et al., 2002; Runov et al., 2003; Sergeev et al., 2003) spacecraft observations of bifurcated current sheets, and allow one to interpret them as the effect of magnetic turbulence. Indeed, for the 29 August crossing, Voros et al. (2003); Runov et al. (2004) estimated a turbulence level of $\delta B / B_{0} \sim 0.1$, which is in the range considered here. Our study lends support to the assumption that the bifurcated current sheet is due to current carrying ions, although the electrons could also play a role (see the Introduction).

By varying the anisotropy of the injected distribution function we can reproduce, besides the double current density peak, the diamagnetic (negative) current wings observed by Cluster and by other spacecraft. The diamagnetic current is due to the so-called magnetization current $-c \nabla \times\left(P_{\perp} \mathbf{B} /|\mathbf{B}|\right)$ (Zelenyi et al., 2000). This term is proportional to the perpendicular pressure $P_{\perp}$, that is to $v_{\perp}^{2}$. In the case of weak anisotropy, $v_{\|} \sim v_{\perp}$ and particles give a substantial contribution to the magnetization current. On the other hand, in the case of strong anisotropy, $v_{\|} \gg v_{\perp}$ and the magnetization current term is small (in other words, field-aligned particles spend only a short time at the edges of the current sheet). When the magnetic overshoots are identified in the experimental data, this would give information on the ion velocity distribution, in particular implying weak anisotropy (small streaming velocity $v_{D}$ ). This inference should have influence on the way we think the current sheet is refilled with plasma.

Finally, we note that the double peaked current profile and the magnetic overshoots are observed only sometimes in the data. Their observation by Cluster was related to the largescale flapping motion of the current sheet as a whole, during which the bifurcated structure was rather stable (Sergeev et al., 2003). The origin of such flapping motion is currently under investigation (e.g., Karimabadi et al., 2003), and we note that the flapping motion corresponds to kink modes (Sergeev et al., 2003), which can also be described as bending waves. It was shown by Bertin and Coppi (1985) that large-scale bending waves are excited in a plasma sheet when the current density profile is characterized by strong gradients (although other driving mechanisms are also possible). Those bending waves are characterized by a perturbation amplitude almost independent of $z$, i.e. by a quasi-rigid displacement in the normal direction (Bertin and Coppi, 1985), which corresponds well to Cluster observations of the considered magnetotail crossings. Therefore, we can tentatively suggest the following scenario for the interpretation of Cluster observations: A combination of magnetic turbulence and ion anisotropy leads to the formation of a double peaked current sheet and possibly of diamagnetic wings. The ensuing strong gradient of current density then drives unstable a large-scale bending wave, which causes the flapping motion of the magnetotail current sheet.

Acknowledgements. We would like to acknowledge use of Cluster key parameters on CDAWeb, data provider G. Gustafsson at IRFU. This work was supported by the Italian MIUR (Cofin 2002), by the Center for High-Performance Computing (Centro di Eccellenza MIUR), and by the Research Training Network of the EU "Turbulence in Space Plasmas". L. M. Z. acknowledges the support of RFFI grant 01-02-16637 and of the Humboldt foundation in his the- 
oretical studies. During his stays in Italy A. L. T. was supported by grants of Italian INFM and MIUR.

Topical Editor T. Pulkkinen thanks two referees for their help in evaluating this paper.

\section{References}

Angelopoulos, V., Kennel, C. F., Coroniti, F. V., et al.: Characteristics of ion flow in the quiet state of the inner plasma sheet, Geophys. Res. Lett., 20, 1711, 1993.

Arzner, K. and Scholer, M. : Kinetic structure of the post plasmoid plasma sheet during magnetotail reconnection, J. Geophys. Res., 106, 3827, 2001.

Asano, Y., Mukai, T., Hoshino, M., Saito, Y., Hayakawa, H., and Nagai, T.: Evolution of the thin current sheet in a substorm observed by Geotail, J. Geophys. Res., 108, 1189, 2003.

Bauer, T. M., Baumjohann, W., Treumann, R. A., Sckopke, N., and Lühr, H.: Low-frequency waves in the near-Earth's plasma sheet J. Geophys. Res. 100, 9605, 1995.

Bertin, G. and Coppi, B.: Bending waves and current disk model for the heliosphere, Astrophys. J., 298, 387-399, 1985.

Borovsky, J. E., Elphic, R. C., Funsten, H. O.and Thomsen, M. F.: The Earth's plasma sheet as a laboratory for flow turbulence in high- $\beta$ MHD, J. Plasma Phys., 57, 1, 1997.

Büchner, J. and Zelenyi, L. M.: Regular and chaotic charged particle motion in magnetotaillike filed reversals, 1. Basic theory of trapped motion, J. Geophys. Res., 94, 11 821, 1989.

Delcourt, D. C. and Belmont, G.: Ion dynamics at the earthward termination of the magnetotail current sheet, J. Geophys. Res., 103, 4605, 1998.

Greco, A., Taktakishvili, A. L., Zimbardo, G., Veltri, P., and Zelenyi, L. M.: Ion dynamics in the near-Earth magnetotail: magnetic turbulence versus normal component of the average magnetic field, J. Geophys. Res., 107, 115, 2002.

Hesse, M., Winske, D., Kuznetsova, M. M., Birn, J., and Schindler, K.: Hybrid modeling of the formation of thin current sheets in magnetotail configurations, J. Geomagn. Geoelectr., 48, 749, 1996.

Holland, D. L. and Chen, J.: Self-consistent current sheet structures in the quiet-time magnetotail, Geophys. Res. Lett., 20, 1775, 1993.

Hoshino, M., Nishida, A., Yamamoto, T., and Kokubun, S.: Turbulent magnetic field in the distant magnetotail: Bottom-up process of plasmoid formation?, Geophys. Res. Lett., 21, 2935, 1994.

Hoshino, M., Nishida, A., Mukai, T., Saito, Y., Yamamoto, T., and S. Kokubun, Structure of plasma sheet in magnetotail: Doublepeaked electric current sheet, J. Geophys. Res., 101, 24775 , 1996.

Karimabadi, H., Pritchett, P. L., Daughton, W., and KraussVarban, D.: Ion-ion kink instability in the magnetotail: 2. Three-dimensional full particle and hybrid simulations and comparison with observations, J. Geophys. Res., 108, 1401, doi:10.1029/2003JA010109, 2003.

Kropotkin, A. P., Malova, H. V., and Sitnov, M. I.: Self-consistent structure of a thin anisotropic current sheet, J. Geophys. Res., 102, 22 099, 1997.

Malara, F., Veltri, P. and Carbone, V.: Competition among nonlinear effects in tearing instability saturation., Phys. Fluids B, 4, 3070, 1992.

Malova, H., Bykov, A. A., Popov, V. Yu., Pulkkinen, T. M., Sharma, A. S., and Zelenyi, L. M.: Structure of non-adiabatic current sheets: role of the trapped population and phase mixing, in Proceedings of the Fifth International Conference on Substorms, St. Petersburg, 16-20 May, ESA SP-443, 177-182, 2000.

Nakamura, R., Baumjohann, W., Runov, A., Volwerk, M., Zhang, T. L., Klecker, B., Bogdanova, Y., Roux, A., Balogh, A., Rème, H., Sauvaud, J. A., and Frey, H. U.: Fast flow during current sheet thinning, Geophys. Res. Lett. 29, 55, 2002.

Ohtani, S., Higuchi, T., Lui, A. T. Y., and Takahashi, K.: Magnetic fluctuations associated with tail current disruption: fractal analysis, J. Geophys. Res. 100, 19 135, 1995.

Runov, A., Nakamura, R., Baumjohann, W., et al.: Cluster observation of a bifurcated current sheet, Geophys. Res. Lett., 30 doi:10.1029/2002GL016136, 2003.

Runov, A., Sergeev, V., Nakamura, R., Baumjohann, W., et al.: Properties of bifurcated current sheet observed on August 29, 2001, Annales Geophys., in press, 2004.

Sergeev, V. A., Mitchell, D. G., Russel, C. T., and Williams, D. J.: $\quad$ Structure of the tail plasma/current sheet at $\sim 11 R_{E}$ and its changes in the course of a substorm, J. Geophys. Res., 98, $17345,1993$.

Sergeev, V. A., Runov, A., Baumjohann, W., et al.: Current sheet flapping motion and structure observed by Cluster, Geophys. Res. Lett., 30 doi:10.1029/2002GL016500, 2003.

Sitnov, M. I., Zelenyi, L. M., Malova, H. V., and Sharma, A. S.: Thin current sheet embedded within a thicker plasma sheet: selfconsistent kinetic theory, J. Geophys. Res., 105, 13 029, 2000.

Sitnov, M. I., Guzdar, P. N., and Swisdak, M.: A model of the bifurcated current sheet, Geophys. Res. Lett., 30, 1712, 2003.

Slavin, J. A., Smith, E. J., Sibeck, D. G., Baker, D. N., Zwickl, R. D., and Akasofu, S.-I.: An ISEE 3 study of average and substorm conditions in the distant magnetotail, J. Geophys. Res., 90, $10875,1985$.

Veltri, P., Zimbardo, G., Taktakishvili, A. L., and Zelenyi, L. M.: Effect of Magnetic Turbulence on the Ion Dynamics in the Distant Magnetotail, J. Geophys. Res., 103, 14 897, 1998.

Voros, Z., Baumjohann, W., Nakamura, R., Runov, A., et al.: Multiscale magnetic field intermittence in the plasma sheet, Ann. Geophys., , 21, 1955, 2003.

Zelenyi, L. M., Milovanov, A. V., and Zimbardo, G.: Multiscale magnetic structure of the distant tail: self-consistent fractal approach. New Perspectives on the Earth's magnetotail, Geophys. Mon. Ser. 105, AGU, edited by Nishida, A., Baker, D. N., and Cowley, S. W. H., 321, 1998.

Zelenyi, L. M., Sitnov, M. I., Malova, H. V., and Sharma, A. S.: Thin and superthin ion current sheets. Quasi-adiabatic and nonadiabatic models, Nonlin. Proc. Geophys., 7, 127, 2000.

Zelenyi, L. M., Dolgonosov, M. S., Bykov, A. A., Popov, V. Yu., and Malova, Kh. V.: Influence of trapped plasma on the structure of collisionless thin current sheets, Cosmic Research (Engl. Transl.), 40, 357, 2002a.

Zelenyi, L. M., Delcourt, D. C., Malova, Kh. V., and Sharma, A. S.: "Aging" of the magnetotail thin current sheets, Geophys. Res. Lett., 29, 49, 2002b.

Zimbardo, G., Greco, A., Veltri, P., Taktakishvili, A. L., Milovanov, A. V., and Zelenyi, L. M.: Ion dynamics in the pre-substorm phase: influence of magnetic turbulence and of the normal component of the magnetic field, in Proceedings of the Fifth International Conference on Substorms, St. Petersburg, 16-20 May, ESA SP-443, 225-228, 2000.

Zimbardo, G., Greco, A., Taktakishvili, A. L., Veltri, P., and Zelenyi, L. M.: Magnetic turbulence and particle dynamics in the Earth's magnetotail, Ann. Geophys., 21, 1947, 2003. 